\title{
Divergência genética entre cultivares de caupi(1)
}

\author{
Francisco José de Oliveira( ${ }^{(2)}$, Clodoaldo José da Anunciação Filho(2), Gerson Quirino Bastos(2) \\ e Odemar Vicente dos Reis ${ }^{(3)}$
}

\begin{abstract}
Resumo - O objetivo deste trabalho foi quantificar a divergência genética de cultivares de caupi, agrupadas por análise multivariada visando à seleção de parentais superiores. Foram utilizadas 16 cultivares de caupi [Vigna unguiculata (L.) Walp.] do banco de germoplasma do Centro de Ciências Agrárias da Universidade Federal do Ceará. As observações fenotípicas foram realizadas num ensaio com delineamento experimental em blocos completos casualizados, com seis blocos e 16 tratamentos, incluindo três testemunhas, com parcela experimental de $24 \mathrm{~m}^{2}$ e área útil de $16 \mathrm{~m}^{2}$, sendo quatro fileiras de plantas, com espaços de 1,0 x 0,5 m, contendo duas plantas por cova. Para mensurar os caracteres fenotípicos, cinco plantas competitivas, localizadas nas duas fileiras centrais da parcela, foram tomadas ao acaso. Os cruzamentos entre os grupos I [TVx-337-3F e Vita-4 (TVu 1977-OD)] e II (Bengala e V-4 Alagoas) podem resultar em produção de novas combinações gênicas, por serem divergentes e reunirem maior número de caracteres agronomicamente desejáveis. Os caracteres que mais contribuem para divergência genética são o comprimento da vagem $(36,80 \%)$ e o peso de 100 sementes $(19,21 \%)$.
\end{abstract}

Termos para indexação: Vigna unguiculata, seleção, genitores, análise multivariada, método de melhoramento.

\section{Genetic divergence among cultivars of cowpea}

Abstract - This work aimed to determine the genetic divergence among cowpea cultivars [Vigna unguiculata (L.) Walp.] when grouped in a multivariate analysis concerning to select superior parents. So 16 cowpea cultivars were used from the germplasm bank of the Centro de Ciências Agrárias of the Universidade Federal do Ceará, in Brazil. The data were accomplished in complete randomized blocks, with six blocks, 16 treatments and three cultivar checks. The total area of experimental plots was $24 \mathrm{~m}^{2}$ and the net area was $16 \mathrm{~m}^{2}$, displayed in four rows, plants were spaced about $1.0 \times 0.5 \mathrm{~m}$ with two plants in each plot. The phenotypic data were estimated from five competitive plants as casual samples on two central rows of each replicate. Breeding among the groups I [TVx-337-3F and Vita-4 (TVu 1977-OD)] and II (Bengala and V-4 Alagoas) may be the direct way to obtain new more productive cultivars because these groups are the most geneticaly divergent. The characters that contributed most to genetic divergence were the medium length of the plants $(36.80 \%)$ and weight of 100 seeds $(19.21 \%)$.

Index terms: Vigna unguiculata, selection, parents, multivariate analysis, breeding methods.

\section{Introdução}

O estudo da diversidade das populações é de grande valia no contexto da evolução das espécies e

(1) Aceito para publicação em 17 de fevereiro de 2003. Extraído da tese de doutorado apresentada pelo primeiro autor à Universidade Federal Rural de Pernambuco (UFRPE), Recife, PE.

(2) UFRPE, Rua D. Manoel de Medeiros, s/n, Dois Irmãos, CEP 52171-900 Recife, PE. E-mail: franseol@uol.com.br clodoaldo-anunciação@bol.com.br, bastosgq@hotmail.com.br

(3) Empresa Pernambucana de Pesquisa Agropecuária, Caixa Postal 1022, CEP 50761-000 Recife, PE. E-mail: ipa@ipa.br na identificação de progenitores divergentes. Tomando-se por base estimativas da divergência genética entre as populações de plantas, é possível inferir sobre a capacidade específica de combinação e a heterose. Assim, antes dos cruzamentos, Falconer (1989) recomenda que sejam efetuadas medidas de divergência genética, as quais auxiliam o melhorista na identificação das combinações mais promissoras, que permitem recuperar linhagens avançadas superiores.

A avaliação da divergência genética tem sido feita por meio de técnicas biométricas, baseadas na quantificação da heterose, ou por processos preditivos, os quais baseiam-se nas diferenças 
morfológicas, fisiológicas e agronômicas dos genitores (Cruz \& Regazzi, 1997). Segundo Machado (1999), a utilização de técnicas multivariadas para estimar a divergência genética tem-se tornado comum entre os melhoristas de plantas. Entre essas técnicas, as mais empregadas são: a análise por componentes principais, quando os dados são obtidos de experimentos sem repetições; a análise por variáveis canônicas, quando os dados são obtidos de experimentos com repetições; e os métodos de agrupamento, cuja aplicação depende da utilização de uma medida de dissimilaridade previamente estimada.

Na quantificação da divergência genética entre os parentais, têm sido utilizadas as técnicas da aglomeração baseadas na distância generalizada de Mahalanobis ( $\left.\mathrm{D}^{2}\right)$, proposta por Mahalanobis (1936), e a técnica de agrupamento pelo método de Tocher (Rao, 1952). Segundo Miranda et al. (1988), Cruz (1990) e Rangel et al. (1991), na seleção de parentais deve-se aliar tanto o bom desempenho dos genótipos quanto as divergências genéticas entre os genótipos.

Por meio das distâncias de Mahalanobis, Bezerra (1997) agrupou em nove classes distintas 32 genótipos de caupi, incluindo linhagens e cultivares precoces de crescimento determinado, de porte ereto e semi-ereto. Entre os genótipos, as variáveis que apresentaram maiores contribuições quanto à divergência genética foram ângulo de inserção dos ramos laterais, número de vagens por planta, comprimento do ramo principal e número de nós no ramo principal.

Outros pesquisadores também estudaram a diversidade genética em caupi, utilizando a estatística $\mathrm{D}^{2}$ de Mahalanobis (Patil \& Bhapkar, 1987; Thiyagarajan et al., 1988; Dharmalingam \& Kadambavanasundaram,1989; Thiyagarajan \& Natarajan, 1989; Renganayaki \& Sree Rengasamy, 1991; Sonaware \& Patil,1991).

O objetivo deste trabalho foi quantificar a divergência genética de cultivares de caupi, agrupadas por análise multivariada visando à seleção de parentais superiores.

\section{Material e Métodos}

Foram utilizadas 16 cultivares de caupi [Vigna unguiculata (L.) Walp.] do banco de germoplasma do Cen- tro de Ciências Agrárias (CCA) da Universidade Federal do Ceará, sendo 11 originárias do Brasil - Bengala e Pitiúba (Pentecoste, Ceará), Branquinho e José do Santo (Boa Viagem, Ceará), CE-525 (Quixadá, Ceará), Sempre verde e Carrapicho (Fortaleza, Ceará), TVu 4369 (Viçosa, Minas Gerais), V-4 Alagoas, V-6 Jaguaribe e V-11 Rubi (Ipean, Pará) - e cinco provenientes do Internacional Institute of Tropical Agriculture, na Nigéria - TVu 476-P2, TVu 2331, TVx-337-3F, Vita-4 (TVu 1977-OD) e S-388.

O delineamento experimental foi em blocos ao acaso, com seis blocos, 16 tratamentos, e três testemunhas (Bengala, Sempre verde e Pitiúba). A parcela experimental foi de $24,0 \mathrm{~m}^{2}$ e a área útil de $16,0 \mathrm{~m}^{2}$, com quatro fileiras de plantas, a intervalos de 1,0 m entre linhas e de $0,5 \mathrm{~m}$ entre covas, com duas plantas por cova após o desbaste. Foram mensurados, em cinco plantas escolhidas ao acaso por parcela, o peso de 100 sementes, o número de sementes/ vagem, o número de vagens/planta, o comprimento da vagem, o comprimento do ramo principal, o número de nós do ramo principal, a área da folha, o número de folhas, o número de ramos laterais e a produção de grãos/planta. Após a obtenção dos valores médios de cada variável por parcela, os dados foram submetidos à análise estatística.

$\mathrm{Na}$ análise da divergência genética entre as 16 cultivares de caupi, avaliadas com relação aos dez caracteres já mencionados, foram empregadas as técnicas multivariadas, conforme Cruz \& Regazzi (1997). Na aplicação da técnica de agrupamento das cultivares, foi adotada a distância generalizada de Mahalanobis (1936), como medida de dissimilaridade e por levar em consideração o grau de dependência entre as variáveis estudadas. Para estimar a distância generalizada de Mahalanobis, inicialmente foram computadas as médias de todas as variáveis de cada cultivar e, em seguida, estabelecida a matriz de covariância residual, a matriz de transformação de dados, a variância das variáveis transformadas, as médias das variáveis não correlacionadas e, finalmente, a técnica de condensação pivotal para resolução da matriz de dispersão.

Com relação ao estabelecimento de grupos similares, foi aplicado o método hierárquico aglomerativo de otimização, proposto por Tocher (Rao, 1952), cujos cálculos foram igualmente baseados na distância generalizada de Mahalanobis $\left(\mathrm{D}^{2}\right)$, admitindo-se que a média das distâncias intragrupo deve ser menor que as distâncias intergrupo (Cruz \& Regazzi, 1997). Além do estudo para formação de grupos, também foram efetuadas as seguintes etapas: investigação da contribuição relativa de cada caráter para a divergência genética entre eles; estimativa da maior distância, dentre o conjunto de menores distâncias, entre cada cultivar; e estimativa das distâncias médias intergrupos correspondentes aos grupos formados. Sub- 
seqüentemente, procedeu-se à elaboração da dispersão gráfica, utilizando os resultados da análise de conglomeração intra e intergrupos entre as cultivares, os quais foram também obtidos com base na estatística $\mathrm{D}^{2}$ de Mahalanobis Nas análises de divergência genética foi utilizado o programa GENES (Cruz, 1997).

\section{Resultados e Discussão}

O máximo valor de divergência genética $\left(D^{2}=253,04\right)$ foi obtido para os pares V-4 Alagoas e TVx-337-3F, de origem geográfica diferente (Brasil e Nigéria, respectivamente), o que pode ter contribuído para a maior dissimilaridade existente entre elas (Tabela 1). Entretanto, os menores valores de $\mathrm{D}^{2}$ foram apresentados pelos pares TVx-337-3F com Vita-4 (TVu 1977-OD) e V-4 Alagoas com Bengala, de mesma origem geográfica, respectivamente, 5,06 e 5,62, significando maior similaridade entre os caracteres considerados. Com relação à distância máxima de $\mathrm{D}^{2}$ obtida entre todas as possíveis combinações de cada uma das cultivares estudadas, observa-se que a maioria apresentou suas respectivas $\mathrm{D}^{2}$ máximas quando combinadas com a cultivar V-4 Alagoas, indicando essa cultivar como a mais divergente no grupo de germoplasma avaliado. Valores máximos de $\mathrm{D}^{2}$ de alta magnitude foram obtidos para as combinações entre a cultivar V-4 Alagoas quando combinada com as cultivares TVu 4369, TVu 2331, Vita-4, S-388 e CE-525 e entre a cultivar TVx-337-3F com as cultivares
Bengala, Pitiúba, José do Santo, Carrapicho, V-6 Jaguaribe, V-11 Rubi, TVu 476-P2 e S-388. Estas combinações merecem considerável atenção na seleção de genótipos para hibridação, principalmente entre V-4 Alagoas com as cultivares TVu 4369, TVu 2331, Vita-4, S-388 e CE-525.

Estes genótipos são, portanto, os mais indicados na combinação híbrida nas etapas iniciais de um programa de melhoramento, esperando-se que, devido à divergência genética, haja uma produção de híbridos de maior efeito heterótico, de maneira a elevar as chances de combinações gênicas favoráveis que permitam a seleção de genótipos superiores. A grande amplitude de $\mathrm{D}^{2}$ e os altos valores estimados para a maioria dos pares de cultivares revelam a grande variabilidade genética existente neste grupo de genótipos, o que torna possível a identificação de genitores para a formação de uma população com uma ampla base genética, aumentando assim, a probabilidade de obtenção de genótipos superiores nas gerações segregantes.

A utilização do método de otimização de Tocher, fundamentado na dissimilaridade, expressa pelas distâncias de Mahalanobis $\left(\mathrm{D}^{2}\right)$, possibilitou a distribuição das cultivares estudadas em seis grupos distintos (Tabela 2). Quanto a procedência das cultivares brasileiras e nigerianas, os resultados indicam que a diversidade geográfica não é, necessariamente, sinônimo de diversidade genética. Resultados

Tabela 1. Estimativas das distâncias generalizadas de Mahalanobis $\left(\mathrm{D}^{2}\right)$ relativas a 16 cultivares de caupi. Recife, PE, $2002^{(1)}$.

\begin{tabular}{|c|c|c|c|c|c|c|c|c|c|c|c|c|c|c|c|c|c|}
\hline Cultivares & B & C & D & E & $\mathrm{F}$ & G & $\mathrm{H}$ & I & $\mathrm{J}$ & $\mathrm{L}$ & M & $\mathrm{N}$ & $\mathrm{O}$ & $\mathrm{P}$ & Q & $\mathrm{D}^{2}>$ & $\mathrm{D}^{2}<$ \\
\hline A & 47,62 & 23,66 & 44,80 & 70,89 & 28,61 & 80,62 & 5,62 & 62,08 & 30,08 & 62,91 & 86,97 & 220,85 & 185,27 & 91,46 & 101,33 & $220,85(\mathrm{~N})$ & $5,62(\mathrm{H})$ \\
\hline B & - & 12,97 & 10,84 & 13,61 & 19,81 & 26,24 & 63,66 & 10,29 & 14,26 & 20,17 & 30,78 & 93,40 & 74,25 & 57,21 & 28,64 & $93,40(\mathrm{~N})$ & 10,29(I) \\
\hline $\mathrm{C}$ & - & - & 16,55 & 23,11 & 13,58 & 34,45 & 35,54 & 24,64 & 9,59 & 30,77 & 31,11 & 110,88 & 84,55 & 69,24 & 49,66 & $110,88(\mathrm{~N})$ & $9,59(\mathrm{~J})$ \\
\hline D & - & - & - & 11,46 & 18,30 & 30,68 & 56,37 & 13,30 & 14,84 & 19,41 & 31,16 & 109,39 & 87,76 & 59,69 & 37,26 & $109,39(\mathrm{~N})$ & $10,84(\mathrm{~B})$ \\
\hline E & - & - & - & - & 28,13 & 22,94 & 87,75 & 17,49 & 25,55 & 21,42 & 22,27 & 71,09 & 53,35 & 50,21 & 24,24 & $87,75(\mathrm{H})$ & 11,46 (D) \\
\hline F & - & - & - & - & - & 32,53 & 45,73 & 34,54 & 8,68 & 40,37 & 36,16 & 122,40 & 93,30 & 73,74 & 51,71 & $122,40(\mathrm{~N})$ & $8,68(\mathrm{~J})$ \\
\hline G & - & - & - & - & - & - & 112,25 & 32,83 & 26,23 & 57,31 & 25,73 & 67,15 & 53,24 & 68,53 & 16,98 & $112,25(\mathrm{H})$ & $16,98(\mathrm{Q})$ \\
\hline $\mathrm{H}$ & - & - & - & - & - & - & - & 82,04 & 51,67 & 75,13 & 105,54 & 253,04 & 215,75 & 114,09 & 132,43 & $253,04(\mathrm{~N})$ & $5,62(\mathrm{~A})$ \\
\hline I & - & - & - & - & - & - & - & - & 15,32 & 9,26 & 52,09 & 119,75 & 97,27 & 35,58 & 23,83 & $119,75(\mathrm{~N})$ & $9,26(\mathrm{~L})$ \\
\hline $\mathrm{J}$ & - & - & - & - & - & - & - & - & - & 28,03 & 40,37 & 123,66 & 96,50 & 57,31 & 40,19 & $123,66(\mathrm{~N})$ & $8,68(\mathrm{~F})$ \\
\hline $\mathrm{L}$ & - & - & - & - & - & - & - & - & - & - & 73,09 & 149,19 & 118,10 & 23,74 & 33,18 & $149,19(\mathrm{~N})$ & $9,26(\mathrm{I})$ \\
\hline M & - & - & - & - & - & - & - & - & - & - & - & 41,25 & 30,19 & 122,67 & 57,37 & $122,67(\mathrm{P})$ & $22,27(\mathrm{E})$ \\
\hline $\mathrm{N}$ & - & - & - & - & - & - & - & - & - & - & - & - & 5,05 & 203,34 & 93,48 & $253,04(\mathrm{H})$ & $5,06(\mathrm{O})$ \\
\hline $\mathrm{O}$ & - & - & - & - & - & - & - & - & - & - & - & - & - & 167,25 & 72,53 & $215,75(\mathrm{H})$ & $5,06(\mathrm{~N})$ \\
\hline $\mathrm{P}$ & - & - & - & - & - & - & - & - & - & - & - & - & - & - & 30,90 & $203,34(\mathrm{~N})$ & $23,74(\mathrm{~L})$ \\
\hline Q & - & - & - & - & - & - & - & - & - & - & - & - & - & - & - & $132,43(\mathrm{H})$ & $16,98(\mathrm{G})$ \\
\hline
\end{tabular}

(1)A: Bengala; B: Sempre verde; C: Pitiúba (testemunhas); D: José do Santo; E: Branquinho; F: Carrapicho; G: TVu 4369; H: V-4 Alagoas; I: V-6 Jaguaribe; J: V-11 Rubi; L: TVu 476-P2; M: TVu 2331; N: TVx-337-3F; O: Vita-4 (TVu 1977-OD); P: S-388; Q: CE-525. 
semelhantes foram obtidos por Thiyagarajan \& Natarajan (1989), Renganayaki \& Sree Rengasamy (1991) e Bezerra (1997). Os grupos I e II, compostos por cultivares de mesma origem, apresentaram as menores medidas de dissimilaridade. Às cultivares integrantes do grupo V e também de mesma origem geográfica, correspondeu a maior distância média $(23,74)$. É importante salientar que as cultivares classificadas são homogêneas dentro do grupo, mas heterogêneas entre grupos.

As distâncias intragrupos são sempre inferiores a qualquer distância intergrupos, atendendo ao critério estabelecido para o método de otimização de Tocher (Rao, 1952; Cruz \& Regazzi, 1997) (Tabela 3). Valores mínimos de $\mathrm{D}^{2}$ foram obtidos entre os grupos III e IV (30,81), I e VI $(35,72)$, III e V $(40,89)$, III e VI $(34,85)$, IV e V $(47,48)$ e IV e VI $(41,55)$, evidenciando que o intercruzamento dos genótipos componentes destes respectivos pares de grupos pode não ser muito indicado para a obtenção de genótipos superiores nas gerações segregantes.

Tabela 2. Agrupamento das 16 cultivares de caupi avaliadas pelo método de Otimização de Tocher, fundamentado na dissimilaridade expressa pela distância de Mahalanobis $\left(D^{2}\right)$. Recife, PE, 2002.

\begin{tabular}{llc}
\hline Grupo Cultivares & Distâncias médias \\
\hline I & TVx-337-3F e Vita-4 (TVu 1977-OD) & 5,06 \\
II & Bengala e V-4 Alagoas & 5,62 \\
III & Carrapicho, V-11 Rubi, Pitiúba, Sempre & \\
& verde, José do Santo, V-6 Jaguaribe e & 16,99 \\
& Branquinho & 16,98 \\
IV & TVu 4369 e CE-525 & 23,74 \\
V & TVu 476-P2 e S-388 & - \\
VI & TVu 2331 & \\
\hline
\end{tabular}

Tabela 3. Distâncias médias dentro e entre grupos correspondentes aos seis grupos formados pelas 16 cultivares de caupi. Recife, PE, 2002(1).

\begin{tabular}{lcccccc}
\hline Grupo $^{(2)}$ & I & II & III & IV & V & VI \\
\hline I & 5,06 & 218,73 & 95,55 & 71,60 & 159,47 & 35,72 \\
II & - & 5,62 & 39,02 & 109,15 & 85,90 & 96,26 \\
III & - & - & 16,99 & 30,81 & 40,89 & 34,85 \\
IV & - & - & - & 16,98 & 47,48 & 41,55 \\
V & - & - & - & - & 23,74 & 97,88 \\
VI & - & - & - & - & - & -
\end{tabular}

${ }^{(1)}$ As distâncias médias dentro dos grupos estão dispostas na diagonal principal e as distâncias médias entre grupos estão dispostas fora da diagonal principal. ${ }^{(2)}$ Grupo I:TVx-337-3F e Vita-4 (TVu 1977-OD); Grupo II: Bengala e V-4 Alagoas; Grupo III: Carrapicho, V-11 Rubi, Pitiúba, Sempre verde, José do Santo, V-6 Jaguaribe e Branquinho; Grupo IV: TVu 4369 e CE-525; Grupo V: TVu 476-P2 e S-388; Grupo VI: TVu 2331.
As maiores distâncias médias foram obtidas entre os grupos I e II $(218,73)$, I e V $(159,47)$ e II e IV $(109,15)$, correspondendo às maiores divergências entre grupos e indicando provavelmente as melhores combinações para cruzamentos. Maluf \& Ferreira (1983) afirmam que a identificação de genótipos superiores com base somente na divergência genética é a estratégia mais adequada para um programa de melhoramento. Neste caso, é mais efetivo realizar cruzamentos entre genótipos altamente divergentes com alto potencial produtivo.

A diversidade genética expressada entre os genótipos componentes dos pares de grupos evidencia-os como parentais em potencial para combinações híbridas, que proporcionariam a formação de uma população com ampla base genética e com maior probabilidade de se obter, nas gerações segregantes, novas combinações gênicas, com características para um alto potencial produtivo. $\mathrm{Na}$ identificação dos parentais mais divergentes sugere-se que sejam selecionados, dentro dos grupos, os parentais com melhores médias em relação aos caracteres que se deseja melhorar, objetivando assim, a máxima concentração de alelos favoráveis, conforme os objetivos da seleção. Neste contexto, Dharmalingam \& Kadambavanasundaram (1989) afirmaram que a seleção de parentais dos grupos mais divergentes pode ser feita com base nas médias dos caracteres que tenham maior influência na produção.

Em relação aos resultados apresentados pelos pares I (TVx-337-3F e Vita-4) com II (Bengala e V-4 Alagoas); I (TVx-337-3F e Vita-4) com V (TVu 476-P2 e S-388); e II (Bengala e V-4 Alagoas) com IV (TVu 4369 e CE-525), considerados os mais divergentes, espera-se que o cruzamento intergrupos de cada par de genótipos propicie maior variabilidade nas populações segregantes. No entanto, as médias intragrupos (Tabela 4) apresentadas para o caráter peso médio de 100 sementes e comprimento médio da vagem pelo grupo I, número médio de vagens/planta e produção média de grãos/planta pelo grupo II, número de sementes/vagem e número de vagens/planta pelo grupo III, comprimento médio da vagem e número de vagens/planta pelo grupo IV, estão abaixo dos valores médios destes caracteres (Tabela 5), fato que diminui a possibilidade de obtenção de recombinantes com alto potencial produtivo. 
Os caracteres que mais contribuíram para a diversidade genética foram o comprimento da vagem $(36,87 \%)$, o peso de 100 sementes $(19,21 \%)$ e o número de sementes/vagem $(9,62 \%)$ (Tabela 6). Estes resultados indicam a existência de variabilidade genética significativa para estes caracteres nas cultivares estudadas. As variáveis que menos contribuíram para a divergência genética detectada nesse germoplasma foram o peso de grãos por planta $(0,35 \%)$, o número de nós no ramo principal $(1,62 \%)$ e o número de vagens/planta $(3,89 \%)$. Os estudos de divergência genética aplicada em caupi têm mostrado resultados discordantes quanto à contribuição de cada componente para a diversidade. De acordo com Thiyagarajan et al. (1988), o peso de 100 sementes e o comprimento do ramo principal foram os caracteres que mais contribuíram para divergência; Thiyagarajan \& Natarajan (1989) reportaram caracteres como o número de vagens por planta, o número de sementes por vagem e a produção de grãos por planta; Renganayaki \& Sree Rengasamy (1991) destacaram o peso de 100 sementes, o comprimento da vagem e a produção de grãos/planta; e Bezerra (1997) registrou as

Tabela 4. Médias intragrupos de cada caráter avaliado em 16 cultivares de caupi. Recife, PE, 2002.

\begin{tabular}{|c|c|c|c|c|c|c|}
\hline \multirow[t]{2}{*}{ Variáveis } & \multicolumn{6}{|c|}{ Grupo $^{(1)}$} \\
\hline & I & II & III & IV & $\mathrm{V}$ & VI \\
\hline Peso de 100 sementes (g) & 9,67 & 17,93 & 20,68 & 19,16 & 20,29 & 11,77 \\
\hline Número de sementes/vagem & 17,22 & 14,51 & 13,79 & 14,43 & 13,01 & 16,47 \\
\hline Número de vagem/planta & 23,63 & 6,94 & 13,17 & 12,12 & 13,07 & 12,80 \\
\hline Comprimento da vagem $(\mathrm{cm})$ & 13,69 & 26,64 & 23,71 & 15,41 & 16,66 & 19,04 \\
\hline Comprimento do ramo principal $(\mathrm{cm})$ & 114,30 & 137,32 & 115,20 & 114,27 & 79,08 & 104,77 \\
\hline Número de nós no ramo principal & 15,92 & 14,09 & 16,46 & 14,36 & 13,12 & 15,72 \\
\hline Área da folha $\left(\mathrm{cm}^{2}\right)$ & 48,92 & 127,31 & 106,66 & 78,05 & 79,08 & 90,88 \\
\hline Número de folhas/planta & 33,20 & 28,65 & 32,44 & 25,55 & 19,10 & 36,72 \\
\hline Número de ramos laterais & 3,98 & 3,60 & 4,26 & 3,32 & 2,86 & 3,69 \\
\hline Produção de grãos/planta (g) & 32,72 & 26,80 & 32,60 & 35,08 & 32,47 & 25,35 \\
\hline
\end{tabular}

${ }^{(1)}$ Grupo I: TVx-337-3F e Vita-4 (TVu 1977-OD); Grupo II: Bengala e V-4 Alagoas; Grupo III: Carrapicho, V-11 Rubi, Pitiúba, Sempre verde, José do Santo, V-6 Jaguaribe e Branquinho; Grupo IV: TVu 4369 e CE-525; Grupo V: TVu 476-P2 e S-388; Grupo VI: TVu 2331.

Tabela 5. Valores médios de cinco plantas avaliadas em 16 cultivares de caupi, em relação ao peso de 100 sementes (PCS), ao número de sementes/vagem (NSV), ao número de vagem/planta (NVP), ao comprimento da vagem (CV), ao comprimento do ramo principal (CRP), ao número de nós no ramo principal (NNRP), à área da folha (AF), ao número de folhas/planta (NFP), ao número de ramos laterais (NRL) e à produção de grãos/planta (PGP). Recife, PE. 2002 $2^{(1)}$.

\begin{tabular}{|c|c|c|c|c|c|c|c|c|c|c|}
\hline Cultivares & $\begin{array}{c}\text { PCS } \\
(\mathrm{g})\end{array}$ & $\mathrm{NSV}^{(2)}$ & $\mathrm{NVP}^{(2)}$ & $\begin{array}{l}\mathrm{CV} \\
(\mathrm{cm})\end{array}$ & $\begin{array}{l}\text { CRP } \\
(\mathrm{cm})\end{array}$ & $\mathrm{NNRP}^{(2)}$ & $\begin{array}{c}\mathrm{AF} \\
\left(\mathrm{cm}^{2}\right)\end{array}$ & $\mathrm{NFP}^{(2)}$ & $\mathrm{NRL}^{(2)}$ & $\begin{array}{c}\text { PGP } \\
(\mathrm{g})\end{array}$ \\
\hline Bengala & $19,04 a b c$ & $14,43 \mathrm{abcde}$ & $7,85 \mathrm{~cd}$ & $25,73 a$ & $142,97 \mathrm{a}$ & $14,26 \mathrm{ab}$ & $122,58 \mathrm{ab}$ & $30,69 \mathrm{ab}$ & $3,68 \mathrm{ab}$ & $25,67 a b$ \\
\hline Sempre verde & $15,90 \mathrm{bc}$ & 13,93 bcde & $16,36 \mathrm{abc}$ & $19,30 \mathrm{bcd}$ & $83,63 \mathrm{bcd}$ & $12,82 \mathrm{~b}$ & $100,82 \mathrm{abc}$ & 26,42abcde & $4,09 \mathrm{ab}$ & $36,17 \mathrm{ab}$ \\
\hline Pitiúba & $16,17 \mathrm{bc}$ & $14,78 \mathrm{abcd}$ & $12,98 \mathrm{bcd}$ & $22,09 \mathrm{~b}$ & $110,57 \mathrm{abc}$ & $13,76 \mathrm{ab}$ & $82,89 \mathrm{~cd}$ & $27,94 \mathrm{abc}$ & $3,51 \mathrm{ab}$ & $32,54 \mathrm{ab}$ \\
\hline José do Santo & $16,07 \mathrm{bc}$ & 14,27 abcde & $6,22 \mathrm{~d}$ & $20,56 \mathrm{bc}$ & $75,60 \mathrm{~cd}$ & $15,10 \mathrm{ab}$ & $95,11 b c$ & $24,44 \mathrm{bcde}$ & $3,85 \mathrm{ab}$ & $21,50 \mathrm{ab}$ \\
\hline Branquinho & $15,12 \mathrm{~cd}$ & 14,34 abcde & $16,70 \mathrm{abc}$ & $18,20 \mathrm{~cd}$ & $101,27 \mathrm{abcd}$ & $16,30 \mathrm{a}$ & $92,19 b c$ & 27,53abcd & $3,30 \mathrm{ab}$ & $33,81 \mathrm{ab}$ \\
\hline Carrapicho & $15,96 \mathrm{bc}$ & $11,91 \mathrm{e}$ & $7,09 \mathrm{~cd}$ & $20,05 d$ & $133,20 \mathrm{ab}$ & $15,57 \mathrm{ab}$ & $93,19 b c$ & $33,35 \mathrm{ab}$ & $3,85 \mathrm{ab}$ & $18,41 \mathrm{~b}$ \\
\hline TVu 4369 & $18,87 \mathrm{abc}$ & $15,18 \mathrm{babc}$ & $11,63 \mathrm{cde}$ & $16,59 \mathrm{bcd}$ & $129,47 \mathrm{ab}$ & $15,60 \mathrm{ab}$ & 79,97 cde & $30,99 \mathrm{ab}$ & $3,79 \mathrm{ab}$ & $35,92 \mathrm{ab}$ \\
\hline V-4 Alagoas & $16,83 \mathrm{bc}$ & 14,60abcd & $6,04 d$ & $27,56 a$ & $131,67 \mathrm{ab}$ & $13,93 \mathrm{ab}$ & $132,05 \mathrm{a}$ & 26,61abcde & 3,52ab & $27,93 \mathrm{ab}$ \\
\hline V-6 Jaguaribe & $19,41 \mathrm{ab}$ & $13,89 \mathrm{bcde}$ & $14,48 \mathrm{abcd}$ & $19,14 \mathrm{bcd}$ & $54,47 \mathrm{~d}$ & $13,20 \mathrm{ab}$ & 81,40 cde & $24,25 \mathrm{bcde}$ & $3,66 a b$ & $34,86 a b$ \\
\hline V-11 Rubi & $19,35 \mathrm{ab}$ & 13,33 cde & $10,57 \mathrm{~cd}$ & $20,69 b c$ & 104,73abcd & $14,72 \mathrm{ab}$ & 78,42 cde & $32,46 a b$ & $3,93 \mathrm{ab}$ & $25,26 a b$ \\
\hline TVu 476-P2 & $17,74 b c$ & $12,34 \mathrm{de}$ & $13,54 \mathrm{bcd}$ & $19,09 \mathrm{bcd}$ & $59,10 \mathrm{~d}$ & $13,13 \mathrm{ab}$ & $82,02 \mathrm{~cd}$ & $18,08 \mathrm{de}$ & $2,86 \mathrm{~b}$ & $30,71 \mathrm{ab}$ \\
\hline TVu 2331 & $11,77 \mathrm{de}$ & $16,47 \mathrm{ab}$ & $12,80 \mathrm{bcd}$ & $19,04 \mathrm{bcd}$ & $104,77 \mathrm{abcd}$ & $15,72 \mathrm{ab}$ & $90,88 b c$ & $36,72 \mathrm{a}$ & $3,69 \mathrm{ab}$ & $25,35 \mathrm{ab}$ \\
\hline TVx-337-3F & $9,57 \mathrm{e}$ & $16,82 \mathrm{a}$ & $25,76 a$ & $13,61 \mathrm{e}$ & $115,40 \mathrm{abc}$ & $16,38 \mathrm{a}$ & $52,49 \mathrm{de}$ & $34,23 \mathrm{ab}$ & $4,34 \mathrm{a}$ & $37,39 \mathrm{ab}$ \\
\hline Vita-4 (TVu 1977-OD) & $9,77 \mathrm{e}$ & $15,62 \mathrm{abc}$ & $21,50 \mathrm{ab}$ & $13,77 \mathrm{e}$ & $113,20 \mathrm{abc}$ & $15,46 \mathrm{ab}$ & $45,36 \mathrm{e}$ & $32,18 \mathrm{ab}$ & $3,62 \mathrm{ab}$ & $28,05 \mathrm{ab}$ \\
\hline S-388 & $22,84 \mathrm{a}$ & $11,89 \mathrm{e}$ & 14,30abcd & $16,69 \mathrm{de}$ & $103,83 \mathrm{abcd}$ & $13,86 \mathrm{ab}$ & $89,84 b c$ & $17,47 \mathrm{e}$ & $1,74 \mathrm{c}$ & $42,33 a$ \\
\hline CE-525 & $19,45 \mathrm{ab}$ & $13,68 \mathrm{bcde}$ & $12,61 \mathrm{bcd}$ & $14,23 \mathrm{e}$ & $99,07 \mathrm{abcd}$ & $13,12 \mathrm{ab}$ & 76,14 cde & $20,12 \mathrm{cde}$ & $2,86 \mathrm{~b}$ & $34,24 \mathrm{ab}$ \\
\hline Médias & 16,49 & 14,24 & 13,49 & 19,15 & 103,93 & 14,61 & 87,21 & 27,90 & 3,54 & 30,63 \\
\hline CV (\%) & 10,59 & 3,99 & 15,44 & 6,96 & 20,87 & 4,71 & 17,61 & 7,97 & 6,66 & 31,58 \\
\hline
\end{tabular}

${ }^{(1)}$ Médias seguidas por letras distintas diferem entre si a $1 \%$ de probabilidade, pelo teste de Tukey. ${ }^{(2)}$ Análises realizadas com dados transformados em $(\mathrm{x}+0,5)^{0,5}$. 
variáveis número de vagens/planta, comprimento do ramo principal e número de nós no ramo principal como as que mais contribuíram para divergência genética.

O dendrograma da Figura 1 é resultante da análise de conglomeração intra e intergrupos referente às 16 cultivares de caupi, fundamentada nas medidas de dissimilaridade das distâncias de Mahalanobis, agrupadas em seis diferentes classes, tendo como base o método de otimização de Tocher. Os pares V-4 Alagoas e TVx-337-3F, de origem geográfica diferente, apresentaram a maior dissimilaridade existente entre elas. Contudo, os pares TVX-337-3F com Vita-4 e V-4 Alagoas com Bengala, de mesma origem geográfica, exibiram maior similaridade entre os caracteres considerados.

Tabela 6. Contribuição relativa de cada variável de caupi estudada para a divergência genética. Recife, PE, 2002.

\begin{tabular}{lc}
\hline Caráter & Valor obtido (\%) \\
\hline Área da folha & 7,10 \\
Comprimento de ramo principal & 7,48 \\
Comprimento da vagem & 36,87 \\
Número de vagens/planta & 3,89 \\
Número de sementes/vagem & 9,62 \\
Número de nós no ramo principal & 1,62 \\
Número de ramos laterais & 6,04 \\
Número de folhas/planta & 7,84 \\
Peso de 100 sementes & 19,21 \\
Produção de grãos/planta & 0,35 \\
\hline
\end{tabular}

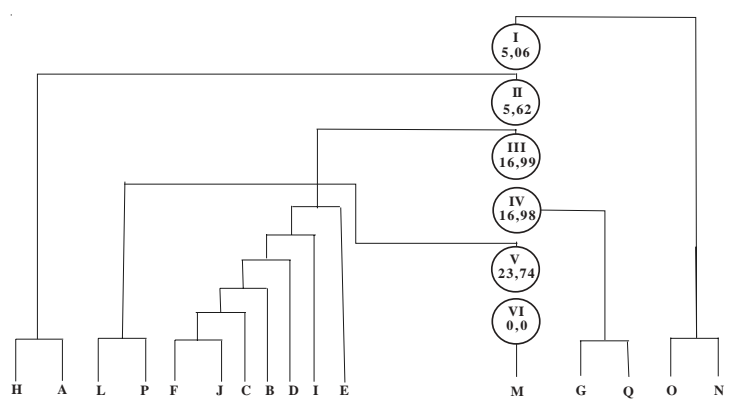

Figura 1. Dendrograma resultante da análise de conglomeração intra e intergrupos em 16 cultivares de caupi, com base na metodologia proposta por Mahalanobis. A: Bengala; B: Sempre verde; C: Pitiúba (testemunhas); D: José do Santo; E: Branquinho; F: Carrapicho; G: TVu 4369; H: V-4 Alagoas; I: V-6 Jaguaribe; J: V-11 Rubi; L: TVu 476-P2; M: TVu 2331; N: TVx-337-3F; O: Vita-4 (TVu 1977-OD); P: S-388; Q: CE-525.

\section{Conclusões}

1. Os cruzamentos entre o grupo I (TVx-337-3F e Vita-4) e o grupo II (Bengala e V-4 Alagoas), podem resultar em produção de novas combinações gênicas, por serem divergentes e reunirem maior número de caracteres agronomicamente desejáveis.

2. Os caracteres comprimento médio da vagem e peso de 100 sementes são os principais determinantes na quantificação da divergência genética entre as cultivares.

\section{Agradecimentos}

Ao técnico Francisco Wellington de Oliveira, da Universidade Federal Rural de Pernambuco, pela colaboração nas diferentes fases de elaboração deste trabalho.

\section{Referências}

BEZERRA, A. A. de C. Variabilidade e diversidade genética em caupi [Vigna unguiculata (L.) Walp.] precoce, de crescimento determinado e porte ereto e semi-ereto. 1997. 105 f. Dissertação (Mestrado em Botânica - Melhoramento Genético de Plantas) - Universidade Federal Rural de Pernambuco, Recife, 1997.

CRUZ, C. D. Aplicação de algumas técnicas multivariadas no melhoramento de plantas. 1990. 188 f. Tese (Doutorado em Agronomia - Melhoramento Genético de Plantas) - Escola Superior de Agricultura Luiz de Queiroz, Piracicaba, 1990.

CRUZ, C. D. Programa GENES: aplicativo computacional em genética e estatística. Viçosa, MG: UFV, 1997. $442 \mathrm{p}$.

CRUZ, C. D.; REGAZZI, A. J. Modelos biométricos aplicados ao melhoramento genético. 2. ed. Viçosa, MG: UFV, 1997. 390 p.

DHARMALINGAM, V.; KADAMBAVANASUNDARAM, M. Genetic divergence in cowpea [Vigna unguiculata (L.) Walp.]. Madras Agricultural Journal, Coimbatore, v. 76, n. 7, p. 394-399, 1989.

FALCONER, D. S. Introduction to quantitative genetics. 3rd ed. New York: Longman, 1989. 489 p.

MACHADO, C. F. Procedimentos para a escolha de genitores de feijão. 1999. 118 f. Dissertação (Mestrado em Agronomia) - Universidade Federal de Lavras, Lavras, 1999. 
MAHALANOBIS, P. C. On the generalized distance in statistics. Proceedings of the National Institute of Sciences of India, New Delhi, v. 2, p. 49-55, 1936.

MALUF, W. R.; FERREIRA, P. E. Análise multivariada da divergência genética em feijão-vagem (Phaseolus vulgaris L.). Horticultura Brasileira, Brasília, v. 1, n. 2, p. 31-34, 1983.

MIRANDA, J. E. C.; CRUZ, C. D.; COSTA, C. P. Predição do comportamento de híbridos e pimentão (Capsicum annuит L.) pela divergência genética dos progenitores. Revista Brasileira de Genética, Ribeirão Preto, v. 11, p. 929-937, 1988 .

PATIL, R. B.; BHAPKAR, D. G. Genetic divergence among 49 cowpea strains. Journal of Maharashtra Agricultural Universities, Rahuri, v. 12, n. 3, p. 283-285, 1987.

RANGEL, P. H. N.; CRUZ, C. D.; VENCOVSKY, R.; FERREIRA, R. P. Selection of local lowland rice cultivars based on multivariate genetic divergence. Revista Brasileira de Genética, Ribeirão Preto, v. 14, n. 2, p. 437-453, 1991.

RAO, R. C. Advanced statistical methods in biometric research. New York: J. Wiley, 1952. 330 p.

RENGANAYAKI, K.; SREE RENGASAMY, S. R. Genetic divergence in vigna species. Indian Journal of Pulses Research, New Delhi, v. 4, n. 2, p. 159-164, 1991.

SONAWARE, M. N.; PATIL, F. B. Genetic divergence in forage cowpea. Journal of Maharashatra Agricultural Universities, Poona, v. 16, n. 2, p. 167-169, 1991.

THIYAGARAJAN, K.; NATARAJAN, C. Genetic divergence in cowpea. Tropical Grain Legume Bulletin, Coimbatore, n. 36, p. 2-3, 1989.

THIYAGARAJAN, K.; RATHINASWAMY, R.; RAJASEKARAM, S. Genetic divergence in cowpea. Madras Agricultural Journal, Pudukottai, v. 75, n. 3/4, p. 125-128, 1988. 